Conclusions $C$ trachomatis screening of pregnant women in the Netherlands is cost-saving.

\section{2-S4.03 THE COST-EFFECTIVENESS OF HUMAN PAPILLOMAVIRUS VACCINATION OF FEMALES OVER AGE 12 YEARS IN THE USA}

doi:10.1136/sextrans-2011-050109.87

H Chesson, L Markowitz. US Centers for Disease Control and Prevention, Atlanta, USA

Background Although the recommended age for Human Papillomavirus (HPV) vaccination of females is 11 to 12 years in the USA, catch-up" vaccination is recommended for females aged 13-26 years who have not been previously vaccinated. The objective of this study was to evaluate the cost-effectiveness of catch-up vaccination strategies for females aged 13-30 years in the USA.

Method We revised and updated a previously-published, spreadsheetbased model of HPV vaccination to estimate the costs and benefits of female HPV vaccination. The health outcomes we included were: cervical intraepithelial neoplasia, genital warts, recurrent respiratory papillomatosis, and HPV associated cancers (cervical, vaginal, vulvar, anal, oropharyngeal, and penile). We examined the cost-effectiveness of catch-up vaccination for three age groups: ages 13-21 years, ages $21-26$ years, and ages 27 to 30 years. We examined a 100 -year time horizon. Routine vaccination of 12 year olds was assumed to occur in all 100 years, with coverage set at $20 \%, 30 \%$, or $75 \%$. The annual probability of receiving catch-up vaccination was $5 \%$ for ages 13 to 18 years and $1.25 \%$ for ages 19 years and older. The duration of the catch-up vaccination program was varied from 1 to 20 years.

Results Catch-up vaccination generally became less cost-effective as routine coverage increased and as the duration of the catch-up program increased. When vaccine coverage and the duration of the catch-up program were varied (and all other parameters were set to their base case values), the incremental cost per OALY gained by extending the duration of catch-up vaccination ranged from $\$ 5000$ to $\$ 40000$ for ages 13 to 21 , from $\$ 50000$ to $\$ 85000$ for ages 21 to 26 , and was $>\$ 140000$ for ages 27 to 30 years. The relatively favourable cost-effectiveness ratios for vaccination of ages 13 to 21 years and the relatively unfavourable cost-effectiveness ratios for vaccination of ages 27 to 30 years were consistent regardless of routine vaccine coverage and the duration of the catch-up vaccine program.

Conclusion Our preliminary findings support the current recommendations of the Advisory Committee on Immunisation Practices (ACIP) for female vaccination. However, although catch-up vaccination for ages 21 to 26 years might be considered cost-effective now, the cost per OALY gained by catch-up vaccination may increase as time goes by and as vaccine coverage increases.

\section{2-S4.04 THE COST OF EXPEDITED PARTNER THERAPY COMPARED TO THE COST OF STANDARD PARTNER REFERRAL FOR THE TREATMENT OF CHLAMYDIA OR GONORRHOEA}

\section{doi:10.1136/sextrans-2011-050109.88}

${ }^{1} \mathrm{~T}$ Gift, ${ }^{2} \mathrm{P}$ Kissinger, ${ }^{3} \mathrm{H}$ Mohammed, ${ }^{1} \mathrm{~J}$ Leichliter, ${ }^{1} \mathrm{M}$ Hogben, ${ }^{4} \mathrm{M}$ Golden. ${ }^{1}$ Centers for Disease Control and Prevention Atlanta, USA; ${ }^{2}$ Tulane University New Orleans, USA; ${ }^{3}$ Ross University School of Veterinary Medicine Basseterre, Saint Kitts and Nevis; ${ }^{4}$ University of Washington Seattle, USA

Background Partner treatment is an important component of sexually transmitted disease (STD) control. Several randomised controlled trials (RCTs) have compared expedited partner treatment (EPT) to unassisted standard partner referral (SR). All of these trials found that EPT significantly increased partner treatment over SR, and some found that EPT significantly lowered re-infection rates in index patients.

Methods We collected cost data to assess the payer-specific, health care system, and societal-level cost of EPT and SR. We used data on partner treatment and index patient re-infection rates from two RCTs examining EPT and SR for patients diagnosed with chlamydia or gonorrhoea. Additional elements were estimated or drawn from the literature, such as the likelihood of progression to and OALY impact of sequelae. We used a Monte Carlo simulation (10000 iterations) to assess the impact on cost and effectiveness of varying several variables simultaneously, and calculated threshold values for selected variables at which EPT and SR costs per patient were equal Sensitivity analyses assessed the impact of varying settings in which EPT might be employed, such as one in which no patient counselling or data entry costs were incurred when employing either EPT or SR. Results From a health care system or societal perspective, EPT was less costly and treated more partners than SR. From the perspective of an individual payer, EPT was less costly than SR if $\geq 40 \%-45 \%$ of male index patients' female partners or $\geq 38 \%$ of female index patients' male partners received care from the same payer. The Abstract O2-S4.04 figure 1 shows the Monte Carlo results for New Orleans and depicts the relationship between the cost difference between EPT and SR and the proportion of partners of the index patient who receive care from the same payer as the index. Negative values in the figure indicate EPT is less costly per patient. In sensitivity analyses, EPT was less costly than SR from all payer perspectives when counselling and data entry costs were eliminated; when counselling costs were applied to EPT alone, the payerperspective cost of EPT was greater than SR for index women, but the additional cost was less than $\$ 2600$ per OALY gained over SR. Conclusions EPT has a lower cost from a societal or health care system perspective than SR and treats more partners. Individual payers may find EPT to be more costly than SR, depending on how many of their patients' partners receive care from the same payer.

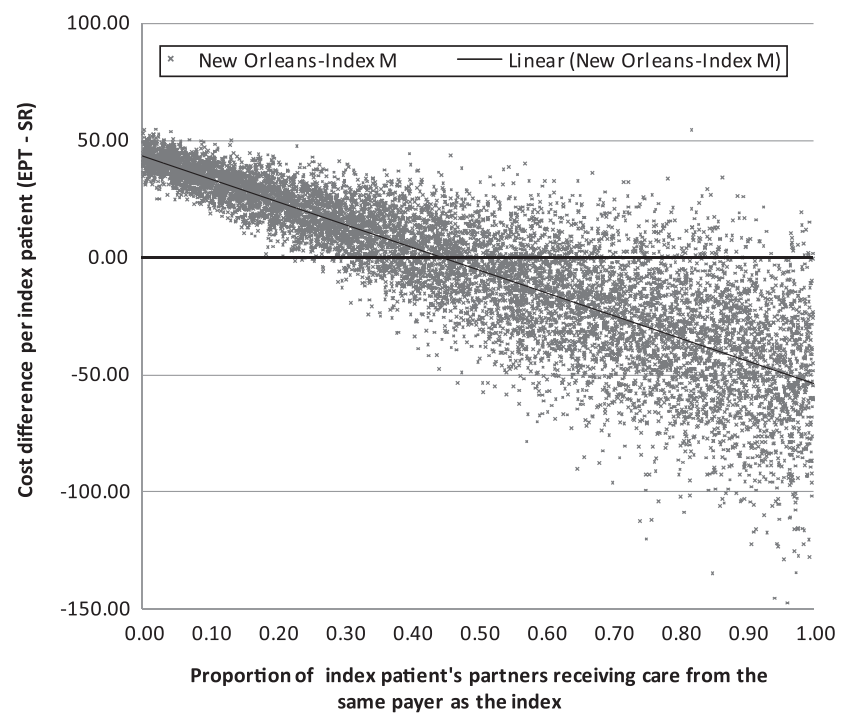

Abstract 02-S4.04 Figure 1 Payer-perspective cost difference per index patient: Expected partner therapy (EPT)-standard referal (SR).

\section{2-S4.05 SEROSORTING BEHAVIOURS AND BELIEFS AMONG MSM AT AN URBAN LGBT HEALTH CENTER}

doi:10.1136/sextrans-2011-050109.89

${ }^{1}$ A Hotton, ${ }^{1} B$ Gratzer, ${ }^{2} \mathrm{D}$ Pohl, ${ }^{3} \mathrm{~S}$ D Mehta. ${ }^{1}$ Howard Brown Health Center, University of Illinois, Chicago School of Public Health, Chicago, USA; ${ }^{2}$ Howard Brown Health Center, Chicago, USA; ${ }^{3}$ University of Illinois, Chicago School of Public Health, Chicago, USA

Background Serosorting, preferentially engaging in unprotected anal intercourse (UAI) with partners of the same HIV status, is practiced by some MSM as a risk reduction strategy. 
Methods May through November 2010, we assessed serosorting practices and beliefs among MSM seeking STI testing at an urban LGBT health center. We compared serosorting practices and beliefs by HIV status and partner characteristics with Pearson $\chi^{2}$ tests. Sexual behaviours, partner characteristics, and HIV status were based on self-report.

Results 705 HIV-negative and 88 HIV-positive MSM completed the assessment. Median time since last HIV test was 7.5 months; nearly a third had not been tested in the last year. Overall, $53 \%$ reported no UAI, $27 \%$ reported seroconcordant UAI, and 18\% reported serodiscordant UAI with last sex partner. UAI was more common with seroconcordant partners than with serodiscordant partners (55\% vs $37 \%, p<0.001) ; 23 \%$ of HIV-positive and $17 \%$ of HIV-negative men reported serodiscordant UAI. $81 \%$ of men with seroconcordant partners said they knew their partner's status by getting tested together or talking with them; $5 \%$ determined partners' status indirectly and $14 \%$ did not report how they determined their partner's status. Seroconcordant UAI was more common among HIVpositive than HIV-negative men ( $53 \%$ vs $24 \%$, p <0.001). Among 288 HIV-negative men reporting UAI: seroconcordant UAI was more common with main vs casual partners $(76 \%$ vs $41 \%, \mathrm{p}<0.001)$ and within monogamous vs non-monogamous relationships (69\% vs $51 \%, p=0.003)$; serodiscordant UAI was more common among men with $\geq 3$ recent sex partners vs $<3$ partners ( $50 \%$ vs $33 \%, p=0.005$ ) and those who reported any anonymous sex partners vs none $(53 \%$ vs $28 \%, \mathrm{p}<0.001)$. Among men who said that they could not be talked out of safer sex with a seroconcordant partner, $10 \%$ of HIVnegative men and $16 \%$ of HIV-positive men reported serodiscordant UAI. $19 \%$ of HIV-negative men who endorsed seroconcordant partnerships reported serodiscordant UAI.

Conclusions Discrepancies between serosorting endorsement and practice underscore the importance of assessing cognitions related to risk-taking and behaviour. High rates of partner concurrency, infrequent testing, and indirect assessment of partner serostatus may limit the effectiveness of serosorting as a risk reduction strategy, even with main partners. The contribution of serosorting to HIV transmission within primary relationships warrants further research.

\section{2-S4.06 EXPLORING ENEMA PRACTICES AMONG MEN WHO HAVE SEX WITH MEN IN THE USA: IMPLICATIONS FOR SEXUAL HEALTH}

doi:10.1136/sextrans-2011-050109.90

\begin{abstract}
${ }^{1} \mathrm{~J}$ G. Rosenberger, ${ }^{1} \mathrm{D}$ Herbenick, ${ }^{1} \mathrm{~B}$ Van Der Pol, ${ }^{2} \mathrm{D} S$ Novak, ${ }^{1} \mathrm{M}$ Reece, ${ }^{3} \mathrm{~J} D$ Fortenberry. ${ }^{1}$ Indiana University, Bloomington, USA; ${ }^{2}$ Online Buddies Inc., Cambridge, USA; ${ }^{3}$ Indiana University School of Medicine, Indianapolis, USA
\end{abstract}

Purpose Recent literature has suggested that use of hygiene products (ie, douching) by women and men prior to sexual activity, changes the biological makeup of vaginal and anal tissue, potentially affecting risk for HIV/STI acquisition. While men who have sex with men (MSM) continue to be disproportionately impacted by HIV/STI, little information exists about men's lifetime enema behaviours, the situational contexts impacting their use, and attitudes towards use of such products.

Methods Data were collected via an internet survey from 27690 18-80 year old MSM. Measures included sociodemographics, recent/ lifetime sexual behaviour history, and items related to enema use.

Results Participants' median age was 39.0 years; ethnicities included white (84.5\%), Latino (6.4\%), African American (3.5\%); and, most $(79.9 \%)$ identified as homosexual. Over half $(54.2 \%)$ of men indicated that they were not currently in a romantic relationship and the majority $(88.9 \%)$ reported having two or more sexual partners in the past year. Most men (64.5\%) reported lifetime enema use, with
$33.8 \%$ reporting use in the past month. Most common reasons for starting enema use were to avoid feces during sex (72.5\%) and to feel clean during sex (65.6\%). Among enema users, $34.4 \%$ reported use of a disposable store bought enema, while $62.5 \%$ indicated having used a refillable enema or one that attached to the shower. Men somewhat or strongly agreed that enema use increased comfort with their anus $(75.8 \%)$, made sex more enjoyable $(75.5 \%)$, and increased the likelihood of being the receptive partner (75.8\%). During the most recent sexual event, enema use was reported both before (37.1\%) and after (8.1\%) sex, with most (42.0\%) reporting use $30 \mathrm{~min}-1 \mathrm{~h}$ prior to sex. Enema use at last sex was more likely to occur among men who were Caucasian $(p<0.01)$, who engaged in anal intercourse $(p<0.01)$, and whose sexual partner was someone they just met $(p<0.05)$.

Conclusions These data provide one of the first examinations of enema practices among MSM in the US. Findings highlight use of enemas prior to sexual activity as a common behaviour, and one associated with positively enhancing the sexual experience. The acceptability of enema use in this population may provide a vehicle for microbicide or virocide delivery once such compounds are fully developed. Future research should examine the relationship between behavioural practices and biological impacts of enema use in relation to HIV/STI.

\section{Social and behavioural aspects of prevention} oral session 5-Individual, Structural and Contextual Coping Strategies: Diverse causes and solutions

\section{2-S5.01 BONDING, BRIDGING, LINKING: EXPLORING RELATIONSHIP BETWEEN SOCIAL CAPITAL AND MIGRANTS' HIV RISK BEHAVIOUR AT DESTINATION}

doi:10.1136/sextrans-2011-050109.91

${ }^{1} \mathrm{D}$ Singh, ${ }^{2} \mathrm{~J}$ Blanchard, ${ }^{3} \mathrm{~J} \mathrm{O}^{\prime} \mathrm{Neil},{ }^{2} \mathrm{~J}$ Mignone, ${ }^{2} \mathrm{~S}$ Moses. ${ }^{1} \mathrm{PHFI}-$ Public Health Foundation of India, New Delhi, India; ${ }^{2}$ University of Manitoba, Winnipeg, Canada; ${ }^{3}$ Simon Frazer University, Canada

Background A multidimensional construct of social capital was employed to understand the relationship between social capital and migrants' HIV risk at destination place.

Methods The study was undertaken among Rajasthani migrants of age 18 and above in Mumbai and Ahmedabad in India to collect data from 1598 migrants through survey method and from 93 migrants through qualitative methods from January to June 2007. Social capital was measured in terms of three domains: bonding, bridging and linking. HIV risk had three measures: having casual partner in last 12 months in the city; sex with a sex worker in the city in last 12 months; and no or inconsistent condom use with a sex worker in last 12 months.

Results Migrants had substantial risk behaviour at destination: 385 respondents $(24.1 \%)$ reported having one or more casual partners; 218 migrants $(13.6 \%)$ had had sex with a sex worker; and 123 respondents $(7.7 \%)$ reported no or inconsistent condom use with a sex worker in the last 12 months in the city. Migrants reported higher risk in Ahmedabad compared to Mumbai for all the three risk measures: 251 (31.6\%) vs 134 (16.7\%) reported casual partners; 138 (17.4\%) vs 80 $(10 \%)$ reported having had sex with a sex worker; and $96(12.1 \%)$ vs $27(3.4 \%)$. Bonding and linking domains of social capital had higher values for migrants in Ahmedabad than Mumbai. Bridging social capital was higher in migrants in Mumbai as compared to migrants in Ahmedabad. All the components of bridging social capital had higher values for migrants in Mumbai than Ahmedabad. Bonding and linking social capital behaved differently in Mumbai and Ahmedabad. In Mumbai, migrants had lower HIV risk if they had high linking social capital and had higher risk if they had high bonding social 